\title{
Towards functional 3D T-ray imaging
}

\author{
Bradley Ferguson ${ }^{1,2,3}$, Shaohong Wang ${ }^{1}$, Doug Gray ${ }^{2,3}$, Derek Abbott ${ }^{2}$ \\ and X-C Zhang ${ }^{1}$ \\ ${ }^{1}$ Department of Physics, Applied Physics and Astronomy, Rensselaer Polytechnic Institute, Troy, \\ NY 12180, USA \\ 2 Centre for Biomedical Engineering and Department of Electrical and Electronic Engineering, \\ The University of Adelaide, SA 5005, Australia \\ ${ }^{3}$ Cooperative Research Centre for Sensor, Signal and Information Processing, Mawson Lakes \\ Boulevard, Mawson Lakes, SA 5095, Australia \\ E-mail: zhangxc@rpi.edu
}

Received 5 March 2002

Published 17 October 2002

Online at stacks.iop.org/PMB/47/3735

\begin{abstract}
We review the recent development of T-ray computed tomography, a terahertz imaging technique that allows the reconstruction of the three-dimensional refractive index profile of weakly scattering objects. Terahertz pulse imaging is used to obtain images of the target at multiple projection angles and the filtered backprojection algorithm enables the reconstruction of the object's frequencydependent refractive index. The application of this technique to a biological bone sample and a plastic test structure is demonstrated. The structure of each target is accurately resolved and the frequency-dependent refractive index is determined. The frequency-dependent information may potentially be used to extract functional information from the target, to uniquely identify different materials or to diagnose medical conditions.
\end{abstract}

\section{Introduction}

Pulsed terahertz (THz) imaging was first proposed by $\mathrm{Hu}$ and Nuss in 1995 (Hu and Nuss 1995). It utilizes the THz, or far-infrared, region of the electromagnetic spectrum and is based upon the technique of terahertz time-domain spectroscopy (THz-TDS). Terahertz imaging has been demonstrated for imaging flames (Mittleman et al 1999), scale-model aircraft (Cheville et al 1997), leaf moisture content (Hadjiloucas et al 1999), skin burn severity, tooth cavities (Ciesla et al 2000) and skin tumours (Loffler et al 2001). One of the primary advantages of $\mathrm{THz}$ imaging is the fact that coherent detection of the broadband pulses provides rich frequency-dependent information on the target's far-infrared optical properties.

Recently, much attention has been focused on three-dimensional (3D) THz imaging techniques. Reflection mode THz tomography systems (Mittleman et al 1997) are based on measuring the time-of-flight of reflected pulses. This technique is capable of resolving 
the $3 \mathrm{D}$ refractive index profile for objects consisting of well-separated layers of differing refractive index. While this technique provides extremely sensitive range resolution of the order of $1 \mu \mathrm{m}$, the current reconstruction algorithms are only applicable given a number of assumptions that restrict its applicability (Mittleman et al 1999). These algorithms neglect multiple reflections, absorption and dispersion in the object to be imaged. These restrictions have motivated research into more general tomographic imaging methodologies.

Other $\mathrm{THz}$ imaging algorithms, drawing inspiration from geophysical (Dorney et al 2001), radar (McClatchey et al 2001) and optical diffraction (Ruffin et al 2001) techniques, are capable of mapping the two- and three-dimensional distribution of scattering objects but have currently only been demonstrated for imaging the shape profile of the target object and both the internal structure and the optical properties of the sample are unavailable.

T-ray computed tomography (T-ray CT) offers the potential to extract the frequencydependent 3D properties of the target (Ferguson et al 2002a). This provides a rich fourdimensional dataset that may be used to uniquely identify materials and may potentially have clinical diagnostic applications. In this paper, we demonstrate the extraction of the frequency-dependent refractive index of a 3D structure.

\section{Methods}

This section describes the hardware and algorithms employed in this work. Section 2.1 describes the chirped probe pulse $\mathrm{THz}$ imaging system, which forms the basis of the 3D T-ray CT system. The chirped probe pulse THz imaging technique can provide a potential speed increase of over a factor of 10 over traditional scanned $\mathrm{THz}$ imaging systems (Hu and Nuss 1995) and does not require $\mathrm{MHz}$ laser repetition rates thus facilitating the use of high power regeneratively amplified lasers. The algorithms employed to reconstruct the frequencydependent refractive index from T-ray CT data are discussed in section 2.2, and section 2.3 describes the experiments that were performed.

\subsection{T-ray imaging with a chirped probe beam}

Traditional THz imaging systems (Hu and Nuss 1995) use a standard THz-TDS system with a $20 \mathrm{~Hz}$ scanning delay line to accelerate the imaging speed. The THz pulse is focused on the sample and the sample scanned in $x$ and $y$ dimensions to form an image. A number of alternative methods have been suggested. A dramatic improvement in acquisition speed was made using two-dimensional electro-optic detection of a collimated $\mathrm{THz}$ pulse (Wu et al 1996).

Electro-optic detection of the $\mathrm{THz}$ radiation with a chirped probe pulse was first demonstrated by Jiang and Zhang (Jiang and Zhang 1998a). This novel technique allows the full $\mathrm{THz}$ waveform to be measured simultaneously rather than requiring a stepped motion stage to scan the temporal profile. This provides a significant reduction in the acquisition time and greatly extends the applicability of $\mathrm{THz}$ systems in situations where the sample is dynamic or moving. Indeed, single shot measurements have been demonstrated measuring the transmitted THz response using a single femtosecond light pulse (Jiang and Zhang 1998b).

The hardware schematic of our imaging system is shown in figure 1. The sample was mounted on a $x-y$ translation stage and raster scanned to acquire an image. THz pulses are generated using a regeneratively amplified Ti:sapphire laser (Spectra Physics Hurricane) with an average output power of $700 \mathrm{~mW}$, a pulse duration of $130 \mathrm{fs}$ and a repetition rate of $1 \mathrm{kHz}$. The pulses are incident on a wide aperture $(16 \mathrm{~mm})$ GaAs photoconductive antenna with a bias of $2000 \mathrm{~V}$. The $\mathrm{THz}$ radiation is focused by off-axis parabolic mirrors to a spot size of 


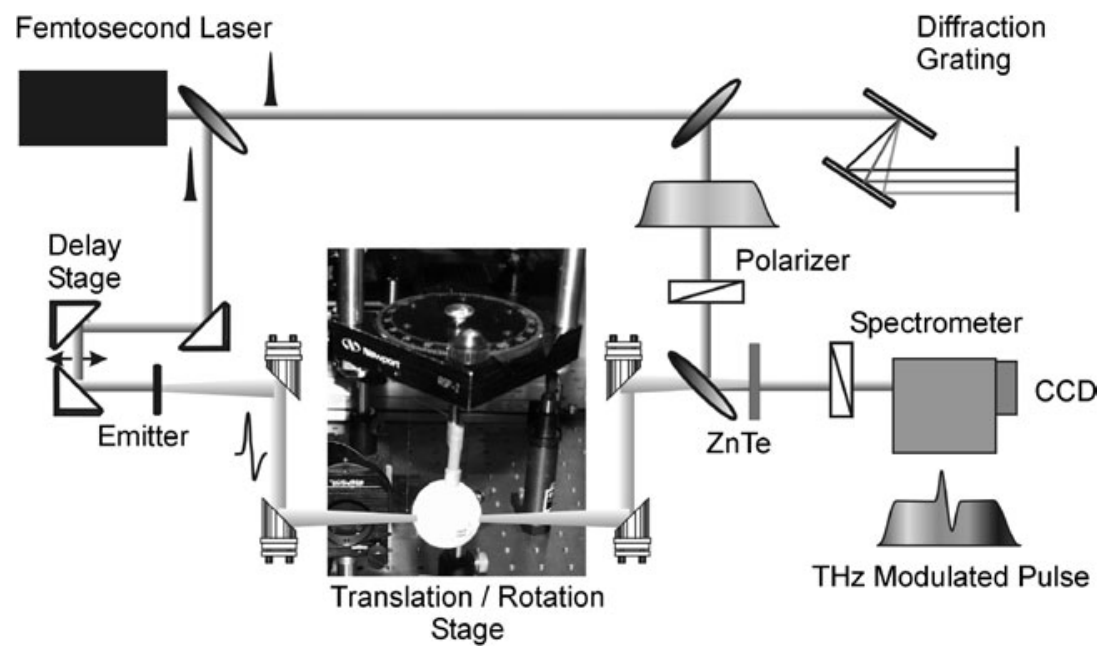

Figure 1. Simplified hardware schematic used for $2 \mathrm{D} \mathrm{THz}$ imaging and T-ray CT. The THz pulse is generated using a femtosecond laser pulse incident on a wide aperture antenna on a GaAs substrate with a bias of $2000 \mathrm{~V}$. The optical probe beam is chirped by a grating pair, this allows the full $\mathrm{THz}$ temporal profile to be sampled simultaneously via electro-optic sampling. A spectrometer and $\mathrm{CCD}$ are used to recover the $\mathrm{THz}$ signal.

approximately $1 \mathrm{~mm}$ on the target. The transmitted $\mathrm{THz}$ pulse is collected with parabolic mirrors and focused onto a $4 \mathrm{~mm}$ thick $\langle 110\rangle$ ZnTe electro-optic (EO) detector crystal.

The optical probe pulse is linearly chirped and temporally stretched to a pulse width of $30 \mathrm{ps}$, using a grating pair with a separation of $4 \mathrm{~mm}$ and pitch of $51^{\circ}$. The THz pulse modulates the probe pulse via the EO effect and is recovered with a spectrometer (SPEX 500M) and CCD camera (PI-Pentamax). The CCD exposure time was set to $50 \mathrm{~ms}$. This provided a signal-to-noise ratio (SNR) of approximately 100. The exposure time could be reduced down to $5 \mathrm{~ms}$ at the expense of SNR. Normal THz-TDS uses a lock-in amplifier for phase sensitive detection of the THz signal. This gives rise to its impressive SNR performance, which may be as high as 10 000:1 (van Exter and Grischkowsky 1990). A lock-in amplifier is not used in chirped probe sampling and this is one of the disadvantages of this technique, although signal processing algorithms may be used to improve the SNR (Ferguson and Abbott 2001). The bandwidth of the system is limited by the chirped pulse detection technique to approximately $1 \mathrm{THz}$. The CCD resolution results in a sampling period of $0.15 \mathrm{ps}$ and a spectral resolution of $17 \mathrm{GHz}$.

\subsection{T-ray computed tomography}

T-ray computed tomography provides sectional images of objects in an analogous manner to conventional CT techniques such as X-ray CT. The filtered backprojection algorithm is then used to reconstruct the target object including both its 3D structure and its frequency-dependent far-infrared optical properties.

Using the hardware system described in section 2.1 , the sample is rotated and a $2 \mathrm{D} \mathrm{THz}$ image is obtained at multiple projection angles. This technique is quite time consuming; a typical image of size $100 \times 100$ pixels measured at 18 projection angles can take over an hour. The imaging speed may be dramatically improved to under a few minutes using $2 \mathrm{D} \mathrm{THz}$ 


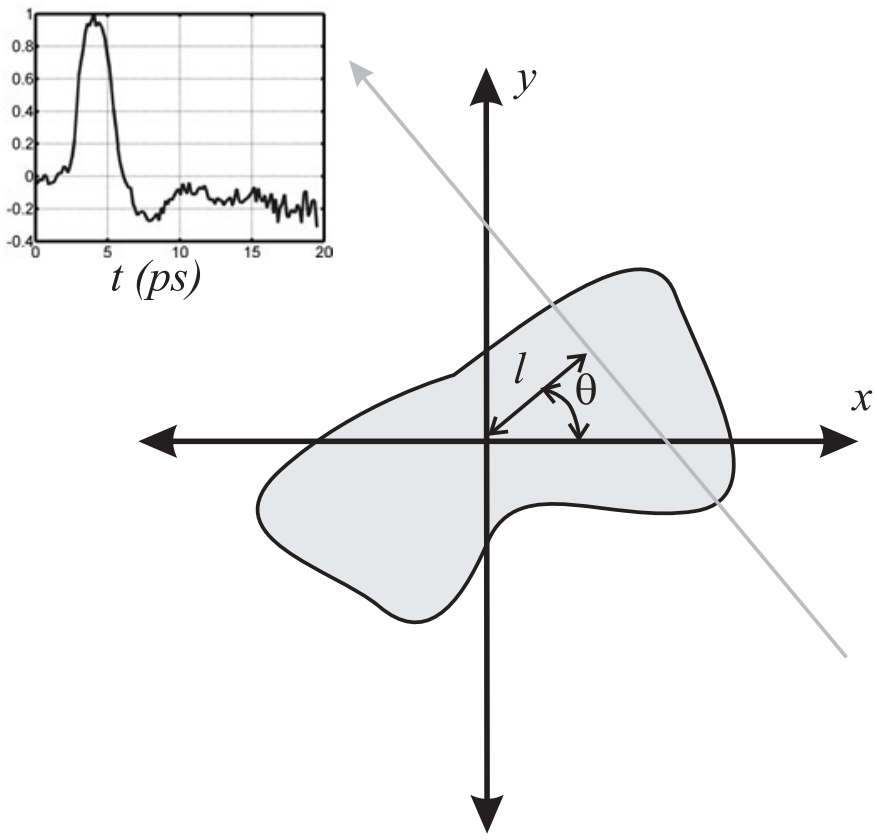

Figure 2. The coordinate system used for T-ray CT. The dimensions $x, y$ and $z$ form the standard Cartesian coordinates ( $z$ is out of the page). The object is rotated about the $z$-axis. The THz beam propagates through the target at an angle $\theta$ to the $x$-axis and offset $l$ from the axis of rotation. The inset shows a typical $\mathrm{THz}$ pulse as a function of time, $t$, after propagation through a target.

imaging with a CCD camera (Wu et al 1996), however, the diffracted $\mathrm{THz}$ radiation then becomes significant and introduces additional processing concerns.

A four-dimensional dataset is acquired in terms of $\{\theta, l, z, t\}$ where $\theta$ is the projection angle, $l$ is the horizontal offset from the axis of rotation, $z$ is the vertical dimension parallel to the axis of rotation and $t$ is the time. We desire a reconstruction of the object optical properties in terms of $\{x, y, z, \omega\}$ where $x, y$ and $z$ are the coordinate axes of the object and $\omega$ is the frequency. The coordinate system is illustrated in figure 2 .

The filtered backprojection algorithm is used to invert the Radon transform to reconstruct the object of interest (Herman 1980). This is based on the approximation that the detected $\mathrm{THz}$ signal is simply related to the incident $\mathrm{THz}$ pulse by a line integral of the form

$$
P_{d}(\omega, \theta, l)=P_{i}(\omega) \exp \left[\int_{L(\theta, l)} \frac{-\mathrm{i} \omega \hat{n}(r)}{c} \mathrm{~d} r\right]
$$

where $P_{d}$ is the Fourier transform of the detected THz signal at a frequency $\omega . \quad P_{i}$ is the Fourier component of the incident THz signal at the same frequency, $c$ is the speed of light, $L$ is the straight line between the source and detector and $\hat{n}(r)=n(r)+\mathrm{i} k(r)$ is the unknown complex refractive index of the sample.

The filtered backprojection algorithm is given by

$$
s(x, y)=\int_{0}^{\pi}\left[\int_{-\infty}^{\infty} S(\theta, \xi)|\xi| \exp [\mathrm{i} 2 \pi \xi l] \mathrm{d} \xi\right] \mathrm{d} \theta
$$

where $S(\theta, \xi)$ is the spatial Fourier transform of the parallel projection data, defined as

$$
S(\theta, \xi)=\int_{-\infty}^{\infty} s(\theta, l) \exp [-\mathrm{i} 2 \pi \xi l] \mathrm{d} l
$$


where $\xi$ is the spatial frequency in the $l$ direction. $s(\theta, l)$ is extracted from the measured projection data. One of the advantages of T-ray CT over X-ray CT is that $s$ may be one of several parameters derived from the $\mathrm{THz}$ pulses. The choice can be made based on the desired application. To perform the reconstruction for the real refractive index $n(\omega)$ at a given frequency, we could use $s=\operatorname{Im}\left\{-\log \left[P_{d}(\omega) / P_{i}(\omega)\right]\right\}$ however this is an oscillatory function and the associated reconstruction is extremely sensitive to noise. A more accurate reconstruction is obtained by using the unwrapped phase of the measured Fourier coefficients such that $s(\theta, l)=\arg \left\{P_{d}(\omega) / P_{t}(\omega)\right\}$, the filtered backprojection algorithm then reconstructs the phase delay at each pixel on the reconstruction grid, $s(x, y)$. The refractive index is recovered according to

$$
n(x, y, \omega)=1+\frac{s(x, y) c}{\omega \mathrm{d} r}
$$

where $\mathrm{d} r=\mathrm{d} x=\mathrm{d} y$ is the reconstruction grid pixel size.

Simpler reconstructions are possible if the sample may be assumed to be non-dispersive. In this case, the bulk refractive index may be reconstructed by choosing $s$ to be the delay of the peak of the THz pulse. Alternatively the bulk absorption coefficient may be reconstructed by using the ratio of the amplitude of the peaks of the detected and incident $\mathrm{THz}$ pulses.

\subsection{Experimental procedure}

Two sample structures were considered: a section of a turkey femur and a plastic structure consisting of a thin plastic cylinder inside a vial. The turkey bone was prepared by soaking it in acetone for $6 \mathrm{~h}$ to ensure maximal transmission of the $\mathrm{THz}$ radiation. Both samples were imaged using a sampling step of $1 \mathrm{~mm}$ in the $x$ and $y$ dimensions to obtain projection images for each $10^{\circ}$ angular increment. The bone structure was then reconstructed using the simple non-dispersive algorithms described in section 2.2. The plastic test structure was reconstructed using the non-dispersive methods and using the frequency-dependent algorithm. The timing of the $\mathrm{THz}$ pulses was used to reconstruct the structure with high accuracy and then the frequency-dependent reconstruction was performed at all available frequencies. This allowed the frequency-dependent refractive index of the inner plastic cylinder to be extracted and independently verified.

\section{Results and discussion}

T-ray CT is restricted in biomedical applications by the severe absorption of THz by moist tissue. However some biological tissues, such as bone, have more moderate absorption coefficients, which makes transmission mode imaging feasible. A section of turkey femur was prepared and imaged as described in section 2.3. A frequency-independent reconstruction was performed using the peak amplitude of the $\mathrm{THz}$ pulses as the input to the filtered backprojection algorithm. Figure 3(a) shows an optical image of the bone sample and figure 3(b) shows a 3D rendered image produced by combining the reconstructed cross sections at each height, $z$. The rendered isosurface threshold was constructed by joining the pixels where the reconstructed absorption coefficient fell to $50 \%$ of the peak absorption coefficient. This resulted in a reconstructed bone diameter of $18 \mathrm{~mm}$ compared to the measured diameter of $22 \mathrm{~mm}$, measured across the widest part of the bone. The irregular surface of the reconstructed image is a result of the surface rendering technique. Variations in the reconstructed absorption as a result of noise or actual variations in the bone's absorption coefficient result in an irregular isosurface. The bone has a fine internal structure that is not accurately reconstructed. This is 


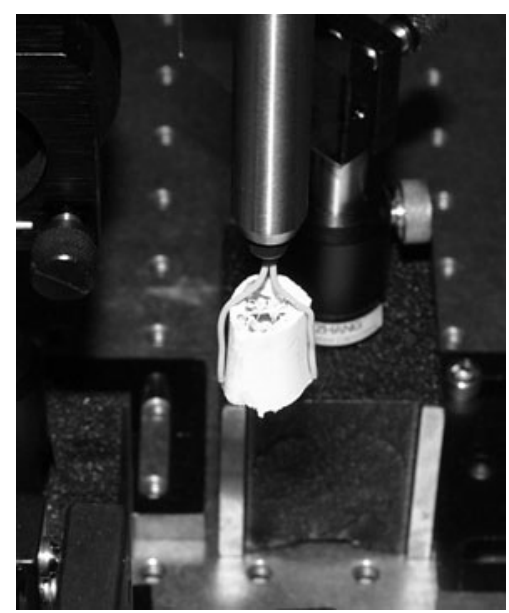

(a)

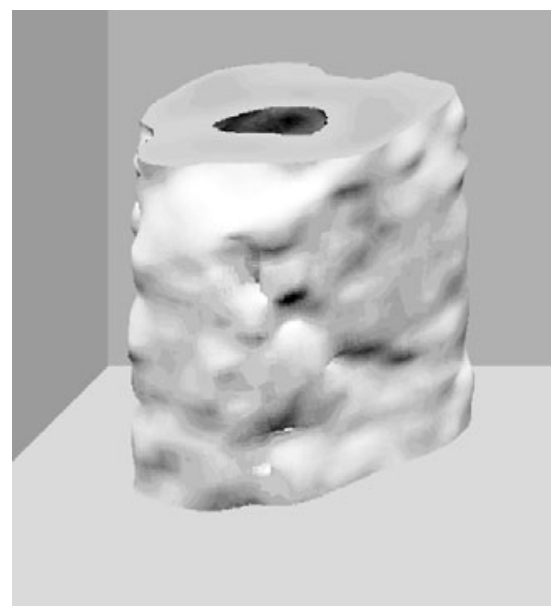

(b)

Figure 3. A section of turkey femur was imaged with the T-ray CT system. The fine structure inside the bone is of the order of the $\mathrm{THz}$ wavelength and therefore causes difficulties in reconstruction. (a) An optical image. The turkey bone was reconstructed and the 3D rendered image generated (b). The reconstruction used the amplitude of the THz pulses at each pixel as the input to the filtered backprojection algorithm.

due to the long wavelength of the THz radiation. Structures with sizes of the order of the wavelength of the $\mathrm{THz}$ radiation cause severe diffraction of the $\mathrm{THz}$ pulse. Diffraction is not accounted for in the reconstruction algorithm and results in artefacts in the final image. Diffraction tomography techniques may be employed to overcome this limitation (Ferguson et al 2002b).

Amplitude reconstructions of the sort shown in figure 3 are useful for revealing structural information, particularly if the internal structure of an object can be revealed. However, a more interesting problem is that of inferring functional information. Recently we have demonstrated classification techniques capable of utilizing the frequency-dependent information provided by $\mathrm{THz}$ imaging to differentiate between different tissue types (Ferguson et al 2001). The use of such algorithms with T-ray CT data promises to enable functional 3D imaging with T-rays. Biomedical imaging with $\mathrm{THz}$ radiation is hindered by the high absorption of most tissues, however, applications may include mapping blood flow and oxidation, or the fat content of tissue. Another promising potential application is in the diagnosis of diseases such as cancer (Loffler et al 2001).

Figure 4(a) shows an optical image of the vial containing a thin plastic tube that was used to demonstrate frequency-dependent 3D reconstruction. First the reconstruction was performed using the timing of the peak of the $\mathrm{THz}$ pulse in the time domain to yield a reconstruction of the bulk refractive index. The reconstruction of the central slice is shown in figure 4(b) and the $3 \mathrm{D}$ rendered image shown in figure 4(c) was produced by combining a number of the reconstructed slices. The isosurface was chosen as the pixels where the reconstructed refractive index was $50 \%$ of the maximum index for the outer vial. The reconstructed image dimensions are quite accurate, the vial and cylinder diameters are within $15 \%$ of the actual dimensions measured with callipers. However, the vial thickness is much thicker than expected because of the coarse reconstruction grid size of $1.5 \mathrm{~mm}$. The grid size may be improved 


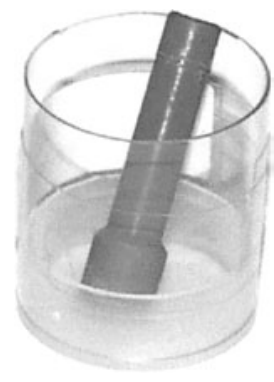

(a)

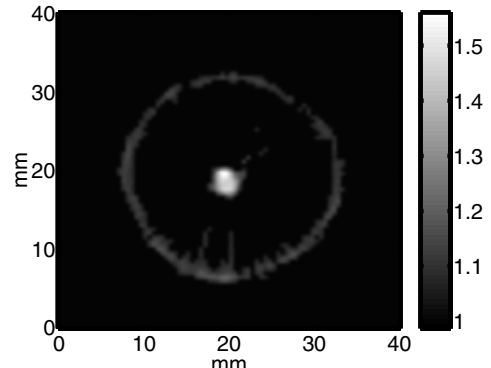

(b)

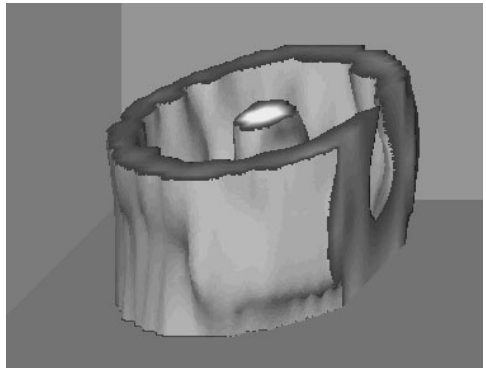

(c)

Figure 4. A vial and plastic tube were used for testing the T-ray CT system. (a) An optical image of the object. The timing of the peak of the pulse in the time domain was used as the input to the filtered backprojection algorithm and the cross section was reconstructed to yield the refractive index as shown in (b). (c) shows a 3D rendered image produced by combining each of the reconstructed cross sections.

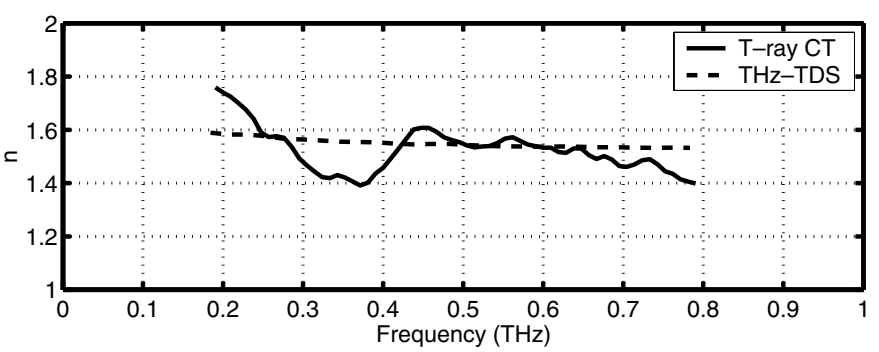

Figure 5. The frequency-dependent refractive index of the plastic inner tube shown in figure 4(a). The refractive index was determined using T-ray $\mathrm{CT}$, which required no assumptions regarding the sample thickness (solid line). The refractive index was also determined with standard THz-TDS using the measured thickness and the algorithm described in (Duvillaret et al 1996) (dashed line). The noisiness of the T-ray CT response is due in part to the low SNR of the chirped probe pulse imaging method compared to THz-TDS.

using more projection angles however the resolution is still limited by the wavelength of the $\mathrm{THz}$ radiation to approximately $1 \mathrm{~mm}$.

The full frequency-dependent reconstruction algorithm described in section 2.2 was then applied. The central slice was reconstructed at each frequency. The pixels corresponding to the inner tube were averaged to yield the refractive index profile shown in figure 5. For reference the frequency-dependent refractive index of the tube was calculated with normal THz-TDS and is also plotted. There is reasonable agreement between the two techniques although the result from T-ray CT is significantly noisier. This is primarily due to the disparate SNRs of the two measurement techniques.

\section{Conclusion}

T-ray CT is a powerful extension of terahertz time-domain spectroscopy with several potential applications. We have demonstrated the ability of T-ray CT to extract the frequency-dependent refractive index of a 3D target thereby providing spectroscopic images of weakly scattering objects. T-ray CT provides the refractive index of the sample without requiring a priori 
knowledge of the sample thickness and allows the internal structure of objects to be revealed. The frequency-dependent reconstruction is noisier than techniques that neglect dispersion and implicitly average the frequency-domain data. However, as the SNR of the T-ray CT hardware is improved it is anticipated that the frequency-dependent information will yield important functional information and enable material classification.

\section{Acknowledgments}

This work was supported by the US Army Research Office, the US National Science Foundation and the Australian Research Council. Bradley Ferguson thanks the AustralianAmerican Fulbright Commission.

\section{References}

Cheville R A, McGowan R W and Grischkowsky D 1997 Late-time target response measured with terahertz impulse ranging IEEE Trans. Antennas Propag. 45 1518-24

Ciesla C M, Arnone D D, Corchia A, Crawley D, Longbottom C, Linfield E H and Pepper M 2000 Biomedical applications of terahertz pulse imaging Proc. SPIE 3934 73-81

Dorney T D, Johnson J L, Rudd J V, Baraniuk R G, Symes W W and Mittleman D M 2001 Terahertz reflection imaging using Kirchhoff migration Opt. Lett. 26 1513-5

Duvillaret L, Garet F and Coutaz J-L 1996 A reliable method for extraction of material parameters in terahertz time-domain spectroscopy IEEE J. Sel. Top. Quantum Electron. 2 739-46

Ferguson B and Abbott D 2001 Wavelet de-noising of optical terahertz pulse imaging data J. Fluctuation Noise Lett. 1 L65-L69

Ferguson B, Wang S, Gray D, Abbott D and Zhang X-C 2001 Terahertz imaging of biological tissue using a chirped probe pulse Proc. SPIE $\mathbf{4 5 9 1} 172-84$

Ferguson B, Wang S, Gray D, Abbott D and Zhang X-C 2002a T-ray computed tomography Opt. Lett. 27 1312-14

Ferguson B, Wang S, Gray D, Abbott D and Zhang X-C 2002b T-ray diffraction tomography OSA Trends in Optics and Photonics (TOPS) The 13th Int. Conf. on Ultrafast Phenomena vol 72 (Washington, DC: Optical Society of America) pp 450-1

Hadjiloucas S, Haratzas L S and Bowen J W 1999 Measurements of leaf water content using terahertz radiation IEEE Trans. Microw. Theory Tech. 47 142-9

Herman G T 1980 Image Reconstruction From Projections—The Fundamentals of Computerized Tomography (New York: Academic)

Hu B B and Nuss M C 1995 Imaging with terahertz waves Opt. Lett. 20 1716-8

Jiang Z and Zhang X-C 1998a Electro-optic measurement of THz field pulses with a chirped optical beam Appl. Phys. Lett. 72 1945-7

Jiang Z and Zhang X-C 1998b Single-shot spatiotemporal terahertz field imaging Opt. Lett. 23 1114-6

Loffler T, Bauer T, Siebert K J, Roskos H G, Fitzgerald A and Czasch S 2001 Terahertz dark-field imaging of biomedical tissue Opt. Express 9 616-21

McClatchey K, Reiten M T and Cheville R A 2001 Time resolved synthetic aperture terahertz impulse imaging Appl. Phys. Lett. 79 4485-7

Mittleman D M, Gupta M, Neelamani R, Baraniuk R G, Rudd J V and Koch M 1999 Recent advances in terahertz imaging Appl. Phys. B 68 1085-94

Mittleman D M, Hunsche S, Boivin L and Nuss M C 1997 T-ray tomography Opt. Lett. 22 904-6

Ruffin A B, Decker J, Sanchez-Palencia L, Le Hors L, Whitaker J F, Norris T B and Rudd J V 2001 Time reversal and object reconstruction with single-cycle pulses Opt. Lett. 26 681-3

van Exter M and Grischkowsky D 1990 Characterization of an optoelectronic terahertz beam system IEEE Trans. Microw. Theory Tech. 38 1684-91

Wu Q, Hewitt T D and Zhang X-C 1996 Two-dimensional electro-optic imaging of terahertz beams Appl. Phys. Lett. 69 1026-8 